\title{
Vorhandlahmheit des Pferdes aus osteopathischer Sicht
}

Ein Bein krank - drei Beine gesund?

Katja Eser

\section{Zusammenfassung}

Die sichtbare Lahmheit oder Taktunreinheit eines Beines lässt noch keinerlei Rückschluss darauf zu, ob tatsächlich lediglich dieses Bein eine Problematik aufweist. Der wichtigste Grundsatz der Osteopathie lautet: Leben ist Bewegung. Insofern interessiert sich die Osteopathie für alle Strukturen, die nicht (mehr) ihre natürliche Motilität, Arthrokinematik und/oder Biomechanik in Qualität bzw. Quantität aufweisen. Betroffen sein können insbesondere Wirbelsegmente, Extremitäten- und Kopf-/Kiefergelenke, Muskeln, Faszien und Organe. Über nervale, fasziale, viszerale und kinetische Verbindungen können der Ort der augenscheinlichen Läsion und die ursächliche Struktur topografisch weit auseinander liegen. Insofern ist es unerlässlich, das Pferd in seiner Gesamtheit zu betrachten und keine voreiligen Schlüsse zu ziehen, die auf einer rein lokalen Befundung fußen.

\section{Was versteht man unter einer Lahmheit?}

Charakteristisch für eine Lahmheit ist ein biomechanisch unphysiologischer Bewegungsablauf mindestens eines Beines in einer Grundgangart, der sich mindestens als Taktunreinheit zeigt. Außer bei einem deutlichen „Hinken“ sind die Taktunreinheiten im Bewegungsablauf häufig in der Ganganalyse nur schwer zu beurteilen. Außerdem geben sie noch keinen abschließenden Aufschluss darüber, ob das Bein, das den unphysiologischen Bewegungsablauf zeigt, auch tatsächlich das „kranke“ Bein ist. Nicht umsonst sind manche tierärztlich durchgeführten Lahmheitsuntersuchungen sowohl zeitlich als auch technisch sehr aufwendig und garantieren trotzdem nicht immer eine belastbare Diagnose. Immer wieder sind die Ergebnisse unbefriedigend: Die Lahmheit ist sichtbar, und man findet einfach keinen Grund dafür. Dies ist für alle Beteiligten ein sehr unzufrieden stellender Zustand: für den Pferdebesitzer, für den Tierarzt und nicht zuletzt für das Pferd, das unter der Lahmheit leidet.

Um der Vorhandlahmheit näher auf die Spur zu kommen, ist zunächst ein Blick auf den natürlichen Bewegungsablauf des Körpers insgesamt und anschließend auf die Biomechanik der Vorhand im Speziellen hilfreich:

\section{Der natürliche Bewegungs- ablauf des Pferdes}

Wie kommt eine physiologische Fortbewegung zustande und wie fließt sie durch den ganzen Körper?

\section{„Der Motor sitzt hinten“}

Der natürliche Bewegungsablauf eines gesunden Pferdes beginnt in der Hinterhand: Das Pferd setzt einen Hinterfuß nach vorne Richtung Schwerpunkt ventral der Rumpfmitte. Der betreffende Hüfthöcker bewegt sich nach ventrokranial. Über die Iliosakralgelenke wird diese Beckenbewegung auf das Sakrum übertragen und setzt sich durch die gesamte Wirbelsäule nach kranial fort.

In der Fortbewegung machen die einzelnen Wirbelkörper eine Lateroflexion (LATFLEX) mit gegensinniger Rotation (ROT; vgl. [1], S.16), sodass der an den Brustwirbeln aufgehängte Brustkorb auf der konkaven Wirbelsäulenseite leicht nach ventral absinkt und sich auf der konvexen Wirbelsäulenseite leicht nach dorsal hebt. Das heißt, der Brustkorb schwenkt dem vorgreifenden Hinterbein aus dem Weg. Diese leichte Brustkorbhebung nimmt das dort aufgelagerte Schulterblatt mit und macht die Vorhand auf der konvexen Seite leicht und frei, um einen Schritt nach vorne zu machen. So weisen denn auch die Grundgangarten Schritt und Trab im biomechanisch gesunden Zustand des Pferdes diagonale Fußfolgen auf (Ausnahme: Passgänge bestimmter Gangpferderassen). Selbst im Galopp, der einzigen Grundgangart, bei der beide Hinterbeine fast zeitgleich nach kranial greifen, findet sich die diagonale Fußfolge.

\section{Die Wirbelsäule als Bogensehnenbrücke}

Die Wirbelsäule des Pferdes funktioniert ähnlich einer Bogensehnenbrücke. Die wichtigsten Bestandteile des ventralen Anteils (die „Sehne“) sind die Pectoralisund Serratusmuskeln, die Abdominalmuskeln mit ihren großflächigen Sehnenanteilen und die Hüftgelenksflexoren. Die wichtigsten Bestandteile des dorsalen Anteils (der „Bogen“) sind die Ligg. nuchae und supraspinale, der M. splenius, der M. erector spinae und die Hüftgelenksextensoren.

Durch Aktivierung der „Sehne“ werden insbesondere die sehnigen Anteile des „Bogens“ gespannt, die Wirbelsäule wölbt sich auf, die Rückenmuskeln entspannen sich, die Hinterhand kommt zum Untertreten und der Rücken kommt zum Schwingen. Im Gegensatz zur Rückenmuskulatur des Menschen, die eine statische Haltefunktion gegen die Schwerkraft hat, ist die Rückenmuskulatur des Pferdes eine dynamisch arbeitende Bewegungsmuskulatur.

\section{Die Vorhand für Balance und Stabilität}

Die Vorhand setzt sich zusammen aus Kopf, Hals, Widerrist und Vorderbeinen. Alleine hieraus wird bereits ersichtlich, dass eine Vorhandlahmheit sehr vielfältige 


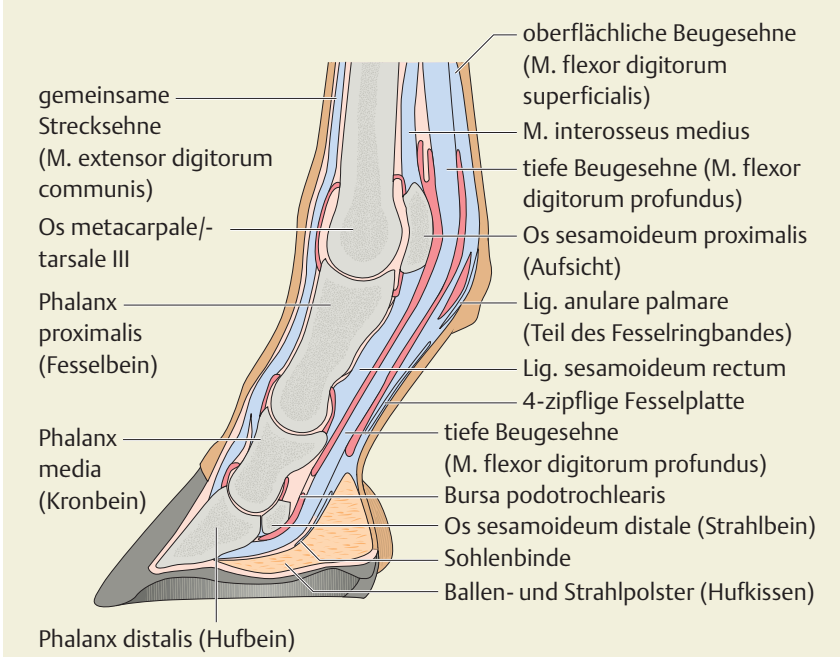

Abb. 1 Sagittalschnitt durch die Vorderzehe. Quellenangaben: Eser K. Checkliste Osteopathie Pferd. Stuttgart: Sonntag in MVS Medizinverlage; 2011.

Ursachen haben kann, die es zu identifizieren gilt.

Hauptfunktionen der Vorderbeine bestehen in der Stabilisierung und Stützung des Rumpfes und der Stoßdämpfung.

\section{Rumpfträger}

Zwischen den Schulterblättern ist der große Brustkorb des Pferdes aufgehängt. Da ihm Schlüsselbeine fehlen, erfolgt beim Pferd diese Aufhängung rein muskulär. Der Vorhand kommt insofern eine ausschlaggebende Funktion der Rumpfstabilität zu, insbesondere durch den M. serratus ventralis thoracis, die Mm. scaleni und die Mm. pectorales (insgesamt als Rumpfträger bezeichnet).

\section{Stoßdämpfung}

Beim Auffußen werden der Druck des Körpers auf den Boden und der Gegendruck des Bodens auf den Körper zunächst im betreffenden Vorderhuf aufgenommen und dann nach dorsal in die Vorhand weitergeleitet.

Als puffernde Strukturen finden sich u.a. (ه Abb. 1):

- die Hufrolle: Bursa podotrochlearis, Insertion des M. flexor digitorum profundus am Strahlbein

- der Fesselträger: M. interosseus medius mit den oberen und unteren Gleichbeinbändern

n zahlreiche Knorpelflächen zwischen den Vorderfußwurzelknöchelchen

- der Rumpfträger (s.o.)

Weitere Hauptaufgabe der Vorderbeine ist es, eine neue Unterstützungsfläche zu finden, wenn der Schub der Hinterhand vor- ne im Pferd ankommt. Im physiologischen Bewegungsablauf ist der Vorgriff der Vorderbeine ein passiver Vorgang, der durch den aktiven Schub der Hinterhand initiiert wird.

\section{Biomechanik der Vorhand}

Der Bewegungsablauf des Vorderbeins lässt sich in 4 Phasen unterteilen: Abfußen, Protraktion, Auffußen und Propulsion.

\section{Abfußen (Hangbein)}

- Flexion des Schulterblatts durch konzentrische Aktivität der Mm. trapezius und rhomboideus Pars thoracis, serratus ventralis thoracis

- Flexion des Schultergelenks durch konzentrische Aktivität der Mm. latissimus dorsi, pectoralis prof., deltoideus und teres major

- Flexion des Ellenbogengelenks durch konzentrische Aktivität der Mm. biceps

- Flexion der Karpal- und Zehengelenke mit Translation des Erbsbeins nach medial durch konzentrische Aktivität der Mm. flexor carpi ulnaris und flexor digitorum superf./prof.

- Gleiten der Gleichbeine nach proximal durch Entspannung des Fesselträgers

- Entlastungsphase des Hufmechanismus:

Entlastung des Strahlpolsters vom Bodendruck

- Anheben der Hufsohle durch Entlastung des Hufbeins

- Zusammenziehen der Hufwand und der Trachten im Bereich der Ballenecken brachii und brachialis n Füllung des proximal des Kornsaums gelegenen Venenplexus nach proximal

- Entlastung des distal des Kronsaums gelegenen Venenplexus

\section{Protraktion (Hangbein)}

- Flexion des Schulterblatts durch konzentrische Aktivität der Mm. trapezius und rhomboideus Pars thoracis, serratus ventralis thoracis

- Extension des Schultergelenks durch konzentrische Aktivität der Mm. brachiocephalicus, cleidobrachialis, pectoralis superf., biceps brachii, supraspinatus

- Flexion des Ellenbogengelenks durch konzentrische Aktivität der Mm. biceps brachii und brachialis

- Extension der Karpal- und Zehengelenke mit Translation des Erbsbeins nach lateral durch konzentrische Aktivität der Mm. extensor digitorum communis und lateralis, extensor carpi radialis

- Gleiten der Gleichbeine nach distal durch Spannung des Fesselträgers

n Extension des zervikothorakalen Übergangs und der BWS durch konzentrische Aktivität des $M$. erector spinae (Mm. spinalis, longissimus, iliocostalis)

\section{Auffußen (Stützbein)}

- Flexion des Schulterblatts durch exzentrische Aktivität des Mm. trapezius und rhomboideus Pars cervicis, serratus ventralis cervicis

- Flexion des Schultergelenks durch exzentrische Aktivität der Mm. brachiocephalicus, supraspinatus und infraspinatus

- Flexion des Ellenbogengelenks durch exzentrische Aktivität des M. triceps brachii

- Extension der Karpal- und Zehengelenke mit Translation des Erbsbeins nach lateral durch konzentrische Aktivität der Mm. extensor digitorum communis und lateralis, extensor carpi ulnaris und exzentrische Aktivität der Mm. flexor digitorum superf./prof.

- Gleiten der Gleichbeine nach distal durch Spannung des Fesselträgers

- Belastungsphase des Hufmechanismus: - erste Abfederung des Bodendrucks durch das Strahlpolster

- Nachgeben der Hufsohle durch Belastung des Hufbeins 
- Dehnung der Hufwand und der Trachten im Bereich der Ballenecken

- Auspressen des proximal des Kornsaums gelegenen Venenplexus nach proximal

- Kompression des distal des Kronsaums gelegenen Venenplexus

\section{Propulsion (Stützbein)}

- Extension des Schulterblatts durch konzentrische Aktivität der Mm. trapezius und rhomboideus Pars cervicis, serratus ventralis cervicis

- Flexion des Schultergelenks durch konzentrische Aktivität der Mm. latissimus dorsi, teres major und minor, deltoideus Pars scapularis, pectoralis prof., Caput longum des M. triceps brachii

- Extension des Ellenbogengelenks durch konzentrische Aktivität des M. triceps brachii

- Extension der Karpal- und Zehengelenke mit Translation des Erbsbeins nach lateral durch konzentrische Aktivität der Mm. extensor digitorum communis und lateralis, extensor carpi radialis

- Gleiten der Gleichbeine nach distal durch Spannung des Fesselträgers

- Flexion des zervikothorakalen Übergangs und der BWS durch konzentrische Aktivität der Mm. obliquus externus und obliquus internus abdominis, rectus abdominis und pectorales

\section{Osteopathische Sicht einer Vorhandlahmheit}

Die Ursachen für Taktunreinheiten und Lahmheiten können sowohl im Bein selbst liegen (s.o. „Biomechanik der Vorhand“) als auch in allen Strukturen, die in Vor-, Mittel- und Hinterhand an der Bewegung beteiligt sind (s.o. „Der natürliche Bewegungsablauf"). Für Definition und Beschreibung der tierärztlich identifizierbaren Lahmheitsursachen sei auf die einschlägige veterinärmedizinische Literatur verwiesen.

Aus osteopathischer Sicht sind die schulmedizinischen Diagnosen im Rahmen der Befundung wissenswerte und wichtige Bausteine, für die Behandlung aber nicht ausschlaggebend. Ausnahme: tierärztliche Kontraindikationen [1] einer osteopathischen Behandlung (Kasten)!

Grundsätzlich wird unterschieden in eine Stützbeinlahmheit und eine Hangbeinlahmheit.
Kontraindikationen einer osteopathischen Behandlung

- absolute Kontraindikationen: Fraktur, Kapsel-/Bänderriss mit Gelenksinstabilität, frischer Muskelriss, frische Blutung, ankylosierte Kissing Spines/Spat, Tumoren, akute fieberhafte Infekte

- relative Kontraindikationen: Trächtigkeit, spinale Ataxie

- Kontraindikationen für kraniosakrale Techniken: frische Kopfverletzungen, Hirntumoren, Gefäßveränderungen im Gehirn, Trächtigkeit in den ersten $3 \mathrm{Mo}$ naten und ab dem 8 . Monat

\section{Stützbeinlahmheit}

Bei der Stützbeinlahmheit ist die Stützbeinphase eines Beines verkürzt. Diese kann sich zeigen als:

- reduzierte Lastaufnahme des Stützbeins

- verfrühtes Abfußen am Ende der Propulsion

Muskuläre Ursachen

in Vorhand und Rumpf

Hypertonus/Kontraktur von (palpierbare Hauptmuskeln):

- M. brachiocephalicus (R. ventralis aus Hirnnerv XI, Rr. ventromediales der Segmente)

- M. pectoralis superficialis (Nn. pectorales craniales und caudales aus nC6Th2)

a M. trapezius Pars thoracis (R. dorsalis aus Hirnnerv XI)

- M. rhomboideus Pars thoracis (Rr. ventromediales der Segmente)

- M. serratus ventralis thoracis (N. thoracis longus aus nC8-nTh1)

- Mm. scaleni (Rr. ventrales der Segmente)

- M. deltoideus Pars clavicularis (N. axillaris aus nC7/nC8)

- M. supraspinatus (N. suprascapularis aus nC6-nC8) (Zügellahmheit)

- M. biceps brachii (N. musculocutaneus aus nC6-nC8)

- M. brachialis (N. musculocutaneus und N. radialis aus nC6-nTh1)

- Mm. flexor digitorum superf./prof. (Nn. ulnaris und medialis aus nC7-nTh2)

- M. extensor carpi ulnaris (N. radialis aus nC7-nTh1)

n M. erector spinae (Rr. dorsales der Segmente)
Segmentale Nervenkompression aufgrund etwaiger Wirbelläsionen mit nachfolgender Hypotonie/Atrophie des entsprechenden Myotoms der Propulsoren.

\section{Osteopathische Ursachen}

in Vorhand und Rumpf

- Wirbel (insbesondere C5-Th10) in EXT oder in LATFLEX/ROT kontralateral

- Scapula in FLEX

- Schultergelenk in EXT

- Ellenbogengelenk in FLEX

- Karpalgelenk in FLEX

- Zehengelenke in FLEX

- Gleichbeine superior

Stolpern des Stützbeins

- Zungenbein (!)

- zervikothorakaler Übergang/Thoraxapertur mit erster Rippe (Cave: Kompression des Plexus brachialis!)

- Th2-Th10

- Atlas/Okziput

- Kiefergelenk/Zähne

- SSB/Dura mater

Organische Ursachen

Mangelhafte Motilität der Organe, z. B. aufgrund von Funktionsstörungen oder Entzündungen, können zu reflektorischen Verspannungen der segmentalen Interkostal- und Intervertebralmuskeln führen, die wiederum eine Blockierung der Facetten-, Rippenkopf- und Rippen-BrustbeinGelenke nach sich ziehen können.
- Lunge
- Herz
- Magen
- Bauchspeicheldrüse
- Leber

Energetische Ursachen

Fülle oder Leere in den Meridianen:

- Herz und Dünndarm

- Lunge und Dickdarm

- Gouverneur und Konzeptionsgefäß

\section{Hangbeinlahmheit}

Bei der Hangbeinlahmheit ist die Spielbeinphase eines Beines verkürzt. Diese kann sich zeigen als:

- verfrühtes Auffußen am Ende der Protraktion

- flacherer oder unharmonischer Bewegungsbogen während der Protraktion 
Muskuläre Ursachen

in Vorhand und Rumpf

Hypertonus/Kontraktur von (palpierbare Hauptmuskeln):

- M. pectoralis profundus (Nn. pectorales craniales und caudales aus nC6-nTh2)

- M. latissimus dorsi (N. thoracodorsalis aus nC8)

- M. serratus ventralis cervicis (Rr. ventromediales der nC4-nC7)

- M. trapezius Pars cervicis (R. dorsalis aus Hirnnerv XI)

- M. rhomboideus Pars cervicis (Rr. ventromediales der Segmente)

- M. deltoideus Pars scapularis (N. axillaris aus $\mathrm{nC} 7, \mathrm{nC} 8$ )

- M. triceps brachii (N. radialis aus nC7nTh1)

- Mm. extensor digitorum communis und lateralis ( $\mathrm{N}$. radialis aus $\mathrm{nC7}-\mathrm{nTh} 1$ )

- M. extensor carpi radialis (N. radialis aus nC7-nTh1)

- Mm. abdomines (Rr. mediales der Segmente)

- Mm. intercostales (Rr. ventrales und Rr. mediales der Segmente)

Segmentale Nervenkompression aufgrund etwaiger Wirbelläsionen mit nachfolgender Hypotonie/Atrophie des entsprechenden Myotoms der Protraktoren.

Osteopathische Ursachen

in Vorhand und Rumpf

- Wirbel (insbesondere C5-Th10) in FLEX oder in LATFLEX/ROT ipsilateral

- Scapula in EXT

- Schultergelenk in FLEX

- Ellenbogengelenk in EXT

- Karpalgelenk in FLEX

- Zehengelenke in EXT

- Gleichbeine inferior

Hinterhandbedingte Ursachen für Hangbeinlahmheit

Eine reduzierte Protraktion eines Hinterbeins führt aufgrund mangelhafter LATFLEX/ROT von Becken, Sakrum und Wirbelsäule (s. o.) zu einem reduzierten Anheben der diagonalen Schulter mit resultierender reduzierter Protraktion dieses diagonalen Vorderbeins.

Protraktion des Hinterbeins (Hangbein):

- Flexion von Becken und LWS durch konzentrische Aktivität des M. iliopsoas

- Flexion des Hüftgelenks durch konzentrische Aktivität der Mm. iliopsoas, gluteus superf., tensor, rectus femoris
- Extension des Kniegelenks durch konzentrische Aktivität des M. quadriceps

- Flexion des Sprunggelenks mit Extension der Zehengelenke durch konzentrische Aktivität der Mm. extensor digitorum longus und lateralis

\section{Organische Ursachen}

Mangelhafte Motilität der Organe, z. B. aufgrund von Funktionsstörungen oder Entzündungen, können zu reflektorischen Verspannungen der segmentalen Interkostal- und Intervertebralmuskeln führen, die wiederum eine Blockierung der Facetten-, Rippenkopf- und Rippen-BrustbeinGelenke nach sich ziehen können.

- Lunge

- Herz

- Magen

- Bauchspeicheldrüse

- Leber

\section{Energetische Ursachen}

Fülle oder Leere in den Meridianen:

n Herz und Dünndarm

- Lunge und Dickdarm

n Gouverneur und Konzeptionsgefäß

Die hier dargestellten möglichen muskulären, osteopathischen, organischen und energetischen Ursachen für eine Vorhandlahmheit erheben nicht den Anspruch auf Vollständigkeit. Jedes einzelne Pferd kann in seinen Strukturen oder seiner Biomechanik individuelle Besonderheiten aufweisen, die den Weg zur Ursache der Lahmheit zeigen.

Um angesichts dieser vielfältigen Strukturen keine potenziell ursächlichen zu übersehen, hat es sich bewährt, die Befundung vom Huf selber nach proximal in die Schulter, dann in die Wirbelsäule nach kranial bis zum Genick und nach kaudal bis in die Hinterhand vorzunehmen.

Aufgrund der großen Bedeutung der physiologischen Rückentätigkeit für eine gesunde Aktivität der Vorhand ist im Rahmen der Behandlung der Vorhandlahmheit neben der lokalen Therapie zusätzlich stets das tägliche Training des Pferdes hinsichtlich Art und Intensität zu überprüfen. Dies dient nicht nur der Prophylaxe von Rezidiven oder Symptomverschiebungen, sondern unterstützt grundlegend die Ausheilung des momentanen Zustands.
() Summary

\section{Osteopathic view of a horse's}

forehand lameness: visible location and structural cause of lesions

If the horse walks, trots or gallops irregularly or is visibly lame in one leg, this does not necessarily mean that it is the concerned leg which is causing the unphysiological movement. The central tenet of osteopathy is that life is movement. Osteopathy examines all structures that do not display (anymore) their natural motility, arthro-kinematics and/ or biomechanics in the normal quantity and/or quality. The vertebral joints, the joints of the extremities and mandibula, muscles, fasciae and organs can be particularly affected. Due to nerval, fascial, visceral and kinetic links across the horse's physiology, there can be big topographical distances between, on the one hand, the region where the lesion is visible and, on the other hand, the region where the actual structure causing the lesion is located. For this reason it is vital to take a comprehensive view and not to draw premature conclusions based on a local examination only.

\section{Key words}

osteopathy - forehand lameness - horse

\section{(- Literatur}

[1] Eser K. Checkliste Osteopathie des Pferdes. Stuttgart: Sonntag Verlag in MVS Medizinverlage Stuttgart; 2011

Online zu finden unter

http://dx.doi.org/10.1055/s-0032-1327798

(- Katja Eser

Judmannstraße 1

85049 Ingolstadt

E-Mail: katja.eser@horsehealing.de

Jahrgang 1967, 1994 Amtsarztprüfung Humanheilpraktikerin, 2002 Staatsexamen Humanphysiotherapie, 2004 Tierheilpraktikerin (ATN), 2006 Pferdeosteopathie (EPOS), 2009 Equine Lymphdrainage nach Prof. Rautenfeld (TiHo Hann.). Seit 1994 in selbstständiger Naturheilpraxis, seit 2002 zusätzlich freiberuflich in Physiotherapiepraxis tätig. Seit 2006 selbstständige Fahrpraxis mit Schwerpunkt Osteopathie und Gymnastizierung für Pferd und Reiter. 2007-2010 Dozentin für Pferdetherapeuten in Ingolstadt. 2011 Veröffentlichung des Buches „Checkliste Osteopathie Pferd“ im Sonntag Verlag 